\title{
Reference Date Time
}

National Cancer Institute

\section{Source}

National Cancer Institute. Reference Date Time. NCI Thesaurus. Code C82518.

The date and time of a specific reference point. 